\title{
Investigation and Research of Smart No-Smoking Reminder
}

\author{
Tong Li* \\ North China Electric Power University (Baoding), Hebei, China \\ *1151859092@qq.com
}

\begin{abstract}
There are 350 million smokers in China and more than 500 million people are injured by second-hand smoke. Nowadays, smoking has become the pain of more and more families. The intelligent anti-smoking supervision system combines anti-smoking reminder devices with APP to achieve intelligent tobacco control and human tobacco control. Our products still advocate physical smoking cessation. Unlike traditional drugs, we are used as a smoking cessation monitoring device to assist users in quitting smoking. No good smoking cessation product can achieve the ultimate effect of smoking cessation without the perseverance of smokers. People who quit smoking need outside supervision, and that's what we offer.
\end{abstract}

Keywords: No Smoking ;reminders; STM32;APP; prompt reminding.

\section{Product Introduction}

The intelligent smoking cessation monitoring device belongs to the innovation project of college students of North China Electric University. This project has designed a new intelligent anti-smoking device. APP can set the alarm threshold for pollutant detection and can set a variety of types of reminders, such as cough, voice reminders, and changing the color of LED lights. APP real-time control of smoke alarm devices, when in a certain space, someone smoking, and we are inconvenient to directly remind, can let the anti-smoking device open, indirectly remind others not to smoke.

The main purpose of the device is to assist family members in quitting smoking. At the same time, when a guest smokes at home, the device can also be "opened" to remind guests not to smoke. This will also protect non-smokers from second-hand smoke at home.

APP settings have two-page modes, one is the above mode to help family members quit smoking, and the other is the mode used by smokers. The combination of these two APP models can achieve more effective anti-smoking effects. This is not only a protection for smokers, but also a reminder of the love of smokers. Smoking is harmful to health. On the road to smoking, we walk hand in hand.

\section{Innovation Point Analysis}

\subsection{The Overall Innovation of the Project}

For the first time, we propose a "soft and hard" approach that combines smart smoking cessation systems with the app in mobile phones. People who quit smoking work with their families to help their families quit smoking.

\subsection{Hardware Module Innovation}

Unlike previous PM2 .5 monitoring devices, our intelligent smoking cessation device can be controlled by APP. PM2 .5 After monitoring the smoke data in the air, the data is transmitted to the 32-chip microcomputer. Our APP can control the setting of the reporting threshold for smoke detection. When the smoke concentration in the air reaches the alarm value, the device automatically alarms. The alarm method can also be set by APP. The basic reminder method is systematic cough and system voice. Users can use their own voice to play according to their own needs. Even if the concentration of smoke in the air has not yet reached the set alarm value, when the user finds someone smoking in the space, he can directly send an instruction with APP to alert the system. 


\subsection{APP Module Innovation Point}

APP is a supporting device for hardware facilities. After it satisfies the function of ordinary smoking cessation APP, it also adds a control system function. Even users who have not yet purchased smart smoking cessation devices are free to download "smoke-free love" APPs in mobile phone stores. They can also visit forums and buy goods. Small APPs can allow users to increase the power to install smart smoking cessation devices while using them. APP can not only assist smart anti-smoking devices, but also promote smart anti-smoking devices.

\section{Project Research}

Analysis 1: In the effective questionnaire, most people have the idea of quitting smoking, indicating that these smokers and their families are the audience for our products; Some people are hesitant about quitting smoking. In the questionnaire survey on the scene, we asked why they hesitated. Most people are unable to find a suitable smoking cessation method, do not have the confidence to quit smoking successfully. These people may also become our potential audience.

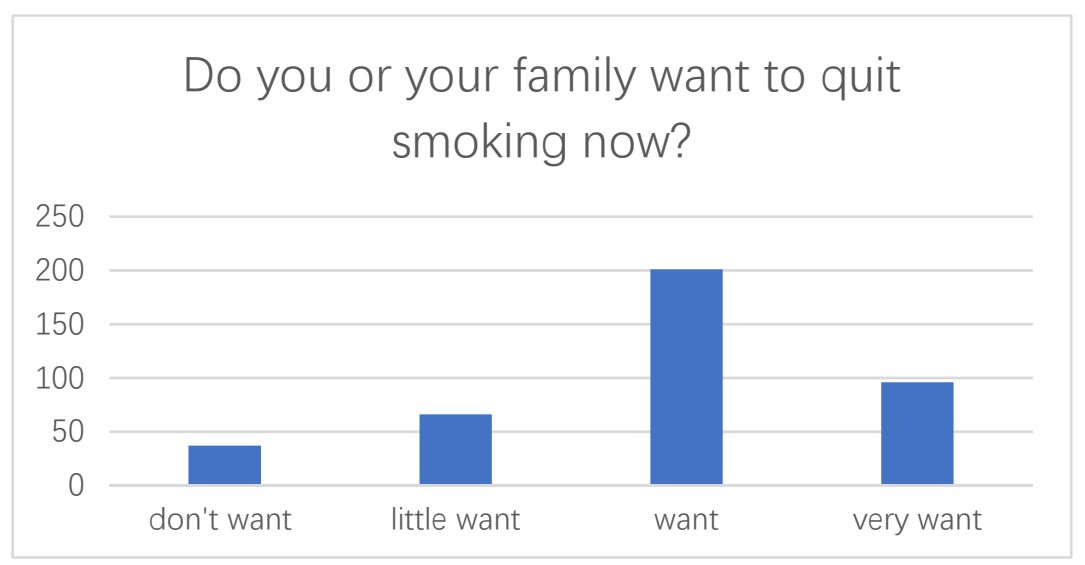

Figure 1. Do you or your family want to quit smoking now?

Analysis 2: The overwhelming majority of the respondents were interested in this new smoking cessation product, but it also depends on the price of the product, so we also need to be accurate about the price of the product.

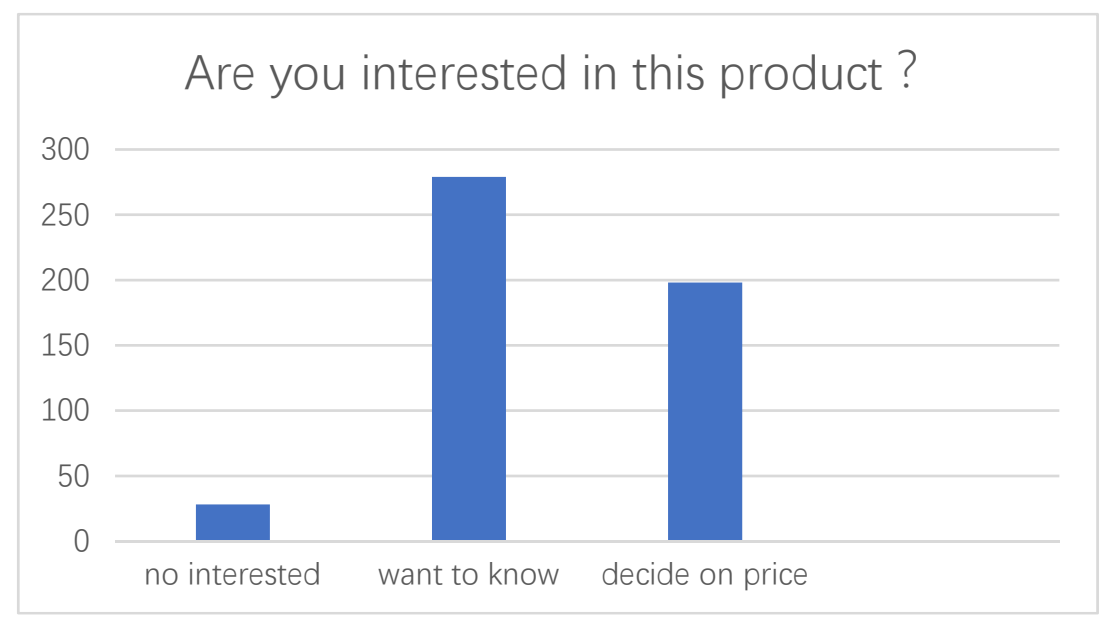

Figure 2. Are you interested in this product?

Analysis 3: The audience's price for the product is about 2,000 yuan. This figure brings us some surprises. The audience's expectations for effective smoking cessation products can be seen. 


\section{In your opinion, the price of the product is somewhat acceptable}

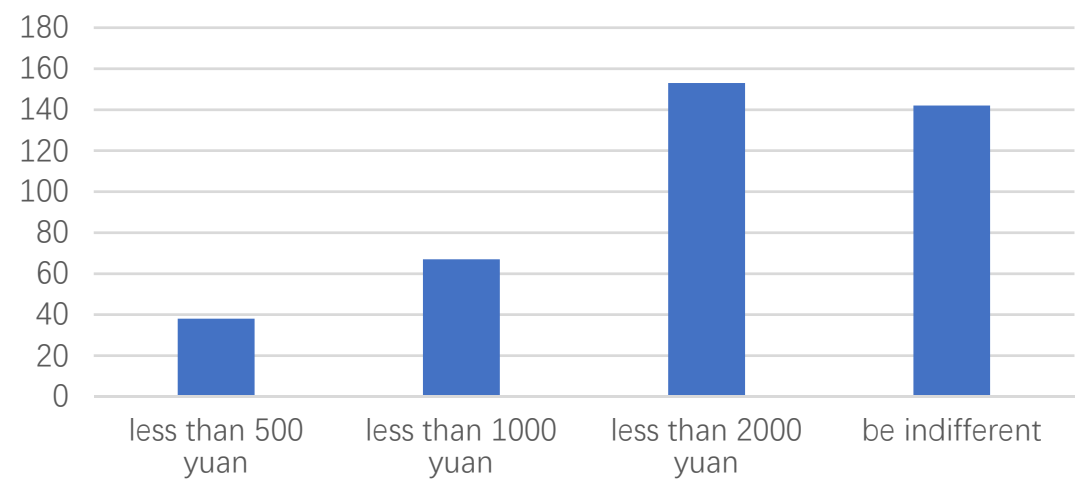

Figure 3. In your opinion, the price of the product is somewhat acceptable

Summary of the Analysis: From this analysis, we learned that there are many people who smoke or have smokers in their homes. This may be our audience. And many of our potential audiences are interested in, and willing to spend money on, such products. This also reflects the audience's expectations of us, so we have to ensure the quality of products, continuously improve the antismoking effect of products.

\section{Promotion Modalities}

In order to make the product known to more people, we decided to use traditional promotion methods and combine new media to promote. Contracted advertisements in the elevator, because the elevator is a closed place, so people inside are more sensitive to cigarettes. It is easy to attract people's attention to hang advertisements for smart anti-smoking systems. Use of new media for publicity. Nowadays, WeChat Public Number has become a media that cannot be ignored. The large WeChat Public Number writes articles and recommends products that will be seen by more people; Many large micro signals write articles recommending products that will attract more people's attention and thus understand our products. the establishment of flagship stores on electronic businesses such as Tmall, JingDong, and Taobao will bring different results. The setting of flagship stores will also allow customers to trust products more. using traditional promotion methods, this advertisement is published in newspapers often read by middle-aged and elderly people and will target a group of audiences; This advertisement is also published in the reading of middle-aged women. It will also target a group of interviewers. In order to achieve the effect of propaganda. The most important one: word of mouth! Product quality is one of the most persuasive points of the product. We need to continuously improve product quality, ensure product effectiveness, so that buyers feel the charm of our smart smoking cessation system, word of mouth publicity, spread 10,10. This is the most ideal publicity effect.

\section{References}

[1]. He Guoxiu, Xuejiaxiu, Zhaowei. Development of Voice Reminder System Based on Single Chip Computer [J]. Intelligent computers and applications.

[2]. Yuanye, Chengshanmei, Huxian. The application of STH32F103 in power electronic control system [J]. Inverter world. 2011(07).

[3]. Elisa K. Tong, Tung T. Nguyen, Eric Vittinghoff, Eliseo J. Pérez-Stable. Smoking Behaviors Among Immigrant Asian Americans. American Journal of Preventive Medicine. 2008 (1). 
[4]. Borland R, Yong H-H, Cummings K M, Hyland A, Anderson S, Fong G T. Determinants and consequences of smoke-free homes: findings from the International Tobacco Control (ITC) Four Country Survey. Tobacco Control. 2006. 